\title{
Adopción de tecnologías agrarias como estrategia para promover la seguridad alimentaria: un estudio en zonas de laderas de Nicaragua
}

\section{Adoption of agricultural technology as a strategy to promote food security: a study in areas of slopes of Nicaragua}

Fidel Guzmán-Guillén', Oswaldo Pineda-Rizo²

${ }^{1}$ Doctor en Planificación y Gestión de Proyectos en Desarrollo Rural Sostenible, Facultad de Desarrollo Rural, fidelblue@gmail.com /2MSc. en Desarrollo Rural, tierrapnd1@gmail.com

Facultad de Desarrollo Rural, (505) 2233-1999 - Tele Fax: 2233-1968

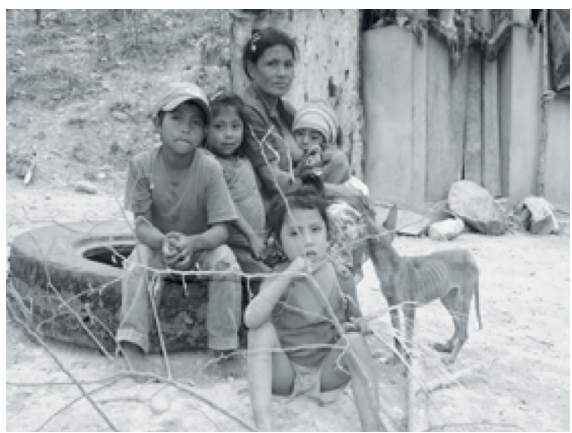

RESUMEN

Las zonas de montaña, cubren la mayor parte de los territorios de Nicaragua y son de gran importancia socioeconómica para el país, ya que la mayoría de los cultivos se establecen en ellas. Los cultivos en laderas y con prácticas tradicionales han causado una degradación severa, lo cual ha agudizado la pobreza rural, y se ha convertido en la prioridad de las metas de las entidades que trabajan en la transferencia de tecnologías de manejo sostenible de suelos en Nicaragua. En esta investigación se plantea el objetivo de analizar la adopción de tecnologías como estrategia para promover la seguridad alimentaria y la conservación del ambiente en la comunidad de Imire en San José de Cusmapa, Madriz; Los Pochotillos, en San Francisco Libre, Managua y en la comunidad de Tomabú, en La Trinidad, Estelí, durante el período 2008-2011, y específicamente, en la determinación de los factores que influyen en la adopción de tecnologías de conservación de suelo, el efecto que han tenido en los rendimientos de los cultivos y en los ingresos económicos de los campesinos para la mejora de su alimentación. La metodología de investigación se fundamenta sobre dos bases complementarias de información: una base secundaria, compuesta por la información generada previamente por otros estudios, publicaciones oficiales, documentación científica y experiencias internacionales, y una base primaria basada en información empírica. Para el registro de los datos de campo se emplean varios instrumentos: entrevistas semi-estructuradas y talleres, involucrando a campesinos y agentes de extensión agraria; además de la socialización de los datos mediante su presentación en talleres productivos. Los resultados muestran que la adopción de técnicas de conservación de suelo es satisfactoria, hay una percepción favorable de las innovaciones que les han sido transferidas. Sin embargo, no logran incrementar los rendimientos de sus cultivos, lo cual no les permite lograr la seguridad alimentaria o mejorar sus condiciones de vida.

Palabras clave: conservación de suelos, calidad de suelo, tecnologías, ingresos productivos, seguridad alimentaria.

Recibido: 29 de enero 2015

Aceptado: 6 de julio 2015

\section{ABSTRACT}

Mountain areas cover most of the Nicaragua's surface and have high socio-economic importance for the country as most of the crops are housed in them. Crops located in slopes and managed with traditional practices have caused a severe degradation of soils, which has exacerbated rural poverty, and have raised concern in the organizations devoted to the Transference of Technology and Sustainable Management of Soils. The goal of this research is analyzing the Adoption of Technologies as a strategy to promote food security and environment preservation in the rural communities San José de Cusmapa Madriz, San Francisco Libre Managua and La Trinidad, Estelí during the period 2008-2011. And specifically in determining the factors that condition the adoption of technologies for the preservation of soils, identifying the effect they have had on crop yields and economic returns of farmers so as to improve their diet. The research technology rests on two bases of complementary information: a secondary basis, comprising information previously generated by other studies, scientific literature, and international experience and a primary basis comprising empirical data. To collect field data several tools are used: semi structured interviews and workshops involving farmers and extension agents; collection of soils samples for lab analysis; socialization of data by their presentation at workshops. Results show that the adoption of soil preservation technologies by the farmers under study is satisfactory and that these technologies result in a better quality of soils and a favorable perception of the innovations that have been transferred. However, they fail to increase the yield of crops, which does not allow them to achieve food security and improve their living conditions.

Keywords: Soil conservation, soil quality, technology, productive income, food security. 


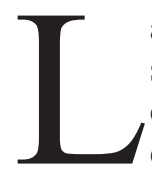

a característica más distintiva de la agricultura es su dinamismo. Las prácticas de cultivo cambian continuamente; sobre la base de su propia experiencia y la de sus vecinos, los agricultores perfeccionan la forma en que manejan sus cultivos. Los cambios de las condiciones naturales, la disponibilidad de recursos y el desarrollo de mercados también plantean retos y oportunidades a los que responden los agricultores. Además, estos se enteran de la existencia de las tecnologías nuevas producidas por diversos organismos, programas, proyectos dedicados a la investigación, la extensión o el desarrollo rural. Estos organismos generan variedades, insumos, prácticas de manejo nuevas y fomentan su empleo, y es esencial que puedan seguir los resultados de sus esfuerzos y comprendan cómo las tecnologías que promueven, encajan en el complejo patrón de cambios en que participan todos los agricultores (CIMMYT, 1993).

El $80 \%$ de los suelos en Nicaragua se encuentran en laderas. Los cultivos alimenticios son producidos por pequeños productores en tierras marginales, por lo que desde la década de los ochenta los organismos públicos y privados, preocupados por el deterioro creciente de los recursos naturales, principalmente el suelo, han promovido tecnologías de conservación de suelos y agua para incrementar la producción, y como alternativa al problema de la erosión del suelo que se da principalmente en zonas de laderas (INTA, 2006).

Es debido a esta característica de los suelos, que uno de los principales problemas en la transferencia de tecnología de manejo sostenible de suelo y agua (MSSA) en Nicaragua, es la falta de indicadores que permitan medir la calidad de su establecimiento, así como la estrategia de comunicación durante el proceso de aceptación y adopción (IICA, 2005).

No obstante, la adopción de técnicas de conservación de suelos y aguas (TCSyA) es el objetivo de muchos organismos que promueven la agricultura sostenible en Nicaragua. Muy a menudo se afirma que esta adopción es lenta y que el proceso de adopción es complejo, sin embargo, no se cuenta con mucha información sobre este proceso (ATyV y PASOLAC, 1999).

Diversas instituciones gubernamentales y no gubernamentales han invertido gran cantidad de recursos económicos, materiales y técnicos en proyectos de transferencia de tecnologías de conservación de suelos y agua en algunos de los municipios y comunidades de nuestro país, donde muy a menudo se encuentra un fenómeno que obstaculiza la factibilidad de dichos proyectos, siendo éste el bajo nivel de adopción de las tecnologías transferidas a los productores que realizan las instituciones. Si las instituciones no cuentan con información que les permita identificar cuáles son esos factores o elementos que dificultan el proceso de adopción, no podrán mejorar sus estrategias de transferencia, pues no sabrían sobre qué elementos deben de incidir, pues estos rara vez reservan fondos para monitorear el progreso, realizando estudios de adopción que permitan conocer la cantidad de agricultores que continúan haciendo las prácticas promovidas o bien han adecuado éstas a sus sistemas de producción cuando el período del proyecto ha concluido (ATy V y PASOLAC,1999).

En la actualidad las instituciones que trabajan en la promoción y transferencia de tecnologías de conservación de suelos y agua, se encuentran con la limitante de no contar con información sistematizada que pueda servir de soporte para mejorar las estrategias de transferencia de estas tecnologías. Debido a esto nace la idea de realizar un estudio que permita identificar cuáles son los factores que determinan el grado de adopción de tecnologías de conservación de suelos y agua transferidas por diferentes instituciones a los productores de la comunidad de Tomabú, municipio de La Trinidad, departamento de Estelí en el periodo 2002-2006.

Con la realización de este estudio se beneficia directamente a las instituciones que laboran en la transferencia de tecnologías de conservación de suelos y aguas, pues podrán conocer cuáles son los factores que determinan el grado de adopción e incorporar dichos resultados a sus estrategias de transferencia maximizando así la posibilidad de éxito del proyecto.

\section{MATERIALES Y MÉTODOS}

\section{Caracterización de la zona}

San José de Cusmapa. Está ubicado entre las coordenadas $13^{\circ} 17^{\prime}$ de latitud norte y $86^{\circ} 39^{\prime}$ de longitud oeste a $248 \mathrm{~km}$ al norte de la capital Managua, y a $34 \mathrm{~km}$ de su cabecera departamental, Somoto. La cabecera municipal se encuentra a 1280 msnm. El municipio de San José de Cusmapa limita al norte con Las Sabanas, al noreste con Honduras, al sur y este con San Juan de Limay y al suroeste con San Francisco del Norte. San José de Cusmapa es la cabecera municipal y se encuentra ubicada a una distancia de $254 \mathrm{~km}$ de Managua. La principal actividad económica es la agricultura y ganadería, considerando al cultivo de café de poca escala; pero también a los granos básicos como maíz, fríjol y sorgo principalmente para el consumo local. La extracción de madera en mediana escala es otra actividad de importancia económica. Según INIDE (2005), cuenta con una población de 7072 habitantes.

La Trinidad. Tiene una extensión territorial de $261 \mathrm{~km} 2$ y se ubica a $18^{\circ} 58^{\prime}$ de latitud norte y $86^{\circ} 14^{\prime}$ de latitud oeste a una altura de $601 \mathrm{msnm}$, comprende una población total de 23882 habitantes, con una distribución demográfica en el sector rural de 13782 habitantes y 10100 en área urbana. El clima del municipio es de tipo tropical seco con poca precipitación pluvial, producto del despale indiscriminado que se ha desarrollado en la zona. La temperatura varía entre los 21 y $25.5^{\circ} \mathrm{C}$. El municipio de La Trinidad se divide en 12 micros regiones que comprenden a su vez un grupo de comarcas y caseríos. La Comunidad de Tomabú pertenece a la Micro región III. 
San Francisco Libre. Se encuentra localizada a $79 \mathrm{~km}$ al noreste de la capital. Presenta una extensión territorial de $756 \mathrm{~km} 2$ y se ubica a $12^{\circ} 30^{\prime}$ de latitud norte y $85^{\circ} 18^{\prime}$ de latitud oeste y a una altura de $40 \mathrm{msnm}$. El municipio posee una población total de 10503 habitantes, con una distribución demográfica en el sector rural de 7423 habitantes y 3080 en área urbana. El clima es de tipo tropical seco en la región baja del pacifico, con una precipitación promedio anual de $1539 \mathrm{~mm}$. La Comunidad de Los Pochotillos está ubicada al noreste del municipio de San Francisco Libre limitando con ciudad Darío. Se encuentra ubicada a aproximadamente a 25 kilómetros del municipio, formado por una extensión de $199.31 \mathrm{~km} 2$. Se caracteriza por la predominancia de pequeños y medianos productores que se dedican a la producción de granos básicos en el siguiente orden de importancia: maíz, sorgo millón y fríjol. Posee una población de 267 habitantes de los cuales 97 son agricultores propietarios con edades entre los 16 y 45 años. (PRESANCA, 2009).

Descripción metodológica. La metodología empleada estuvo compuesta de cuatro grandes fases (a) organización, revisión de fuentes bibliográficas y selección de la muestra (cuadro 1) (b) diseño, validación del instrumento de campo (c) recolección de datos y (d) procesamiento y análisis de datos.

Cuadro 1. Población y muestra de estudio

\begin{tabular}{lccr}
\hline Comunidad & $\begin{array}{c}\text { Total de } \\
\text { productores }\end{array}$ & Muestra & $\%$ \\
\hline Tomabú & 41 & 24 & 23.98 \\
Los Pochotillos & 97 & 34 & 56.73 \\
Imire & 33 & 15 & 19.30 \\
Total & 171 & 73 & 100.00 \\
\hline
\end{tabular}

Variables en estudio. Adopción y seguridad alimentaria y nutricional (SAN) y como sub variables, nivel de escolaridad, tenencia de la tierra, uso de suelo, transferencia tecnológica (capacitación y asistencia técnica) y organización para la variable adopción. En la variable SAN se utilizaron las sub variables disponibilidad (uso actual del suelo e índices productivos), acceso (ingresos económicos productivos /margen bruto), canasta básica y el umbral de reproducción simple.

\section{RESULTADOS Y DISCUSIÓN}

Adopción. Se muestra en el cuadro 2 los 17 tipos de tecnologías de conservación de suelos que han sido ofertadas por diversas instituciones en las comunidades en estudio. En Tomabú se llegó a ofertar hasta 15 tipos de tecnologías, 11 en Los Pochotillos y 12 para el caso Imire.
Cuadro 3. Principales tecnologías adoptadas por productores

\begin{tabular}{lccc}
\hline \multirow{2}{*}{ TCSyA } & \multicolumn{3}{c}{ \% Productores que adoptaron } \\
\cline { 2 - 4 } & Los Pochotillos & Tomabú & Imire \\
\hline Diques & 32 & 7 & 20 \\
Cercas vivas & 47 & 29 & 7 \\
Barreras vivas & 82 & 43 & 93 \\
Barreras muertas & 53 & 50 & 100 \\
\hline
\end{tabular}

En las tres comunidades de estudio se muestra un comportamiento similar cuando de adoptar tecnologías se trata, pues las tres existen mayor cantidad de productores que utilizan barreras vivas y barreras muertas, y en menor grado los diques y las cercas vivas. 
Nivel de escolaridad. Rogers (1995) afirma que es uno de los factores que inciden en la adopción de tecnologías. Estudios expresan que, aquellas personas que presenten mayores dificultades para la apropiación de la información, cuentan con menos herramientas que le permita tomar decisiones en la adopción. Estudios realizados por Ramírez et al., (2000) sobre agricultores de El Salvador y Panamá indican que los productores con educación primaria mostraron tasas de adopción $40 \%$ más altas que quienes no cuentan con educación formal. El hecho de haber terminado los estudios secundarios incrementó las tasas de adopción en otro 30\%. El que los involucrados tuvieran grados educativos relativamente bajos y la presencia recurrente de analfabetismo sugiere que la educación puede ser un factor determinante en la adopción de tecnología. Al respecto Radulovich (1999) afirma que los productores con bajos niveles de educación, tienen mayores problemas para descodificar el mensaje, lo que dificulta que se apropien de la información.

Los resultados obtenidos en este estudio indican que el comportamiento de la adopción no tiene relación con el nivel de escolaridad de los productores. En la figura 2 se aprecia que productores sin nivel de educación lograron adoptar entre seis y 13 tecnologías, y se observa en las tres comunidades que productores con un nivel de educación de primaria adoptan la mayor cantidad de TCSyA.

Tenencia de la tierra. Según Nowak (1992), los productores adoptan nuevas tecnologías por dos razones simples: porque quieren hacerlo y porque pueden hacerlo. No obstante, es claro que el "querer y poder" implementar una innovación, depende entre otras cosas de los recursos tales como la disponibilidad de tierra, tiempo y capital. La

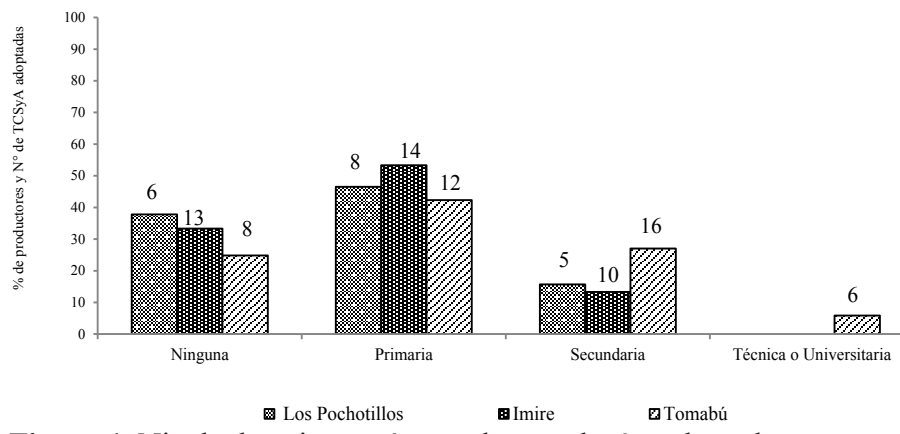

Figura 1. Nivel educativo y número de tecnologías adoptadas por comunidad.

tenencia de la tierra es la relación definida en forma jurídica o consuetudinaria entre personas en cuanto a individuos o grupos, con respecto a la tierra (por razones de comodidad, "tierra" se utiliza aquí para englobar otros recursos naturales, como el agua y los arboles). La tenencia de la tierra es una institución, es decir, un conjunto de normas inventadas por la sociedad para regular el comportamiento. Las reglas sobre la tenencia de la tierra definen de qué manera pueden asignarse dentro de las sociedades los derechos de utilizar, controlar y transferir la tierra, así como, las pertinentes responsabilidades y limitaciones.

Los sistemas de tenencia de la tierra determinan quién puede utilizar qué recursos, durante cuánto tiempo y bajo qué circunstancia. La tenencia de la tierra en las tres zonas de estudio es relativamente similar, ya que el $88 \%$ de los productores de Imire indican que la tierra es propia, en Tomabú el 96\% y en Los Pochotillo el 70\%. Este comportamiento genera condiciones propicias para la adopción de tecnologías y así buscar el aseguramiento de la producción de alimentos para las familias. Los otros sistema de tenencia que se registran en las comunidades corresponden a tierras alquilada, a medias (compartida), prestada, promesa de venta, propia prestada y propia alquilada.

Uso del suelo. En Los Pochotillos e Imire, se registra mayor área destinada a la ganadería, luego el bosque y por último la agricultura, en cambio en Tomabú, se destaca la agricultura y luego la ganadería (figura 2). Este comportamiento de área ganadera en las comunidades de estudio, nos permite identificar las potencialidades en materia de aseguramiento de los alimentos derivados de este rubro.

En las tres comunidades se encontró que los cultivos principales son frijol (Phaseolus vulgaris L.), maíz (Zea mays L.) y sorgo millón (Sorghun bicolor L.), no obstante, también se cultivan tomate (Licopersium sculenta), y Chiltoma (Capsicum annuum L.), pero a muy baja escala. Esto nos refleja lo importante de considerar cualquier acción en materia de transferencia de tecnologías en estas zonas.

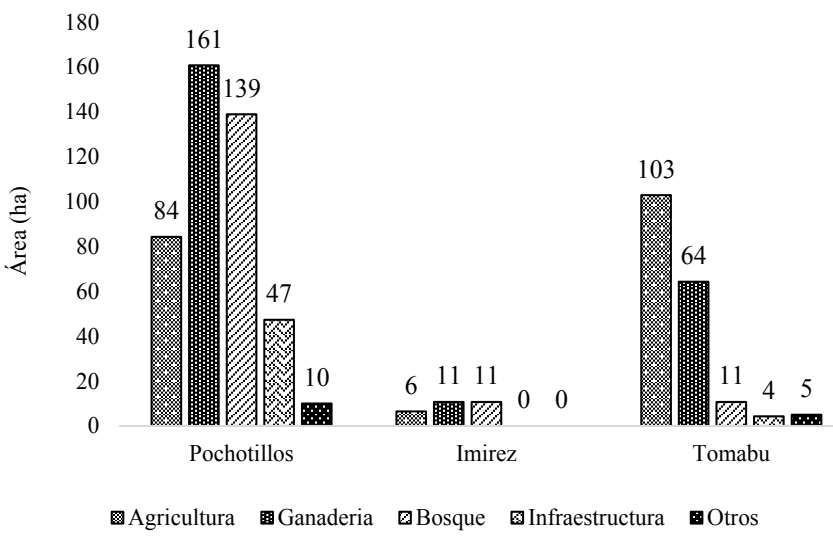

Figura 2. Uso actual del suelo por comunidad.

Área con TCSyA y área total de las fincas. En la figura 3 se observa que Imire se destaca con un mayor porcentaje de TCSyA en relación a Tomabú y Los Pochotillos. Esto se debe fundamentalmente al seguimiento constante e incidencia de las instituciones a nivel local en Imire en materia de conservación de suelo y agua. 


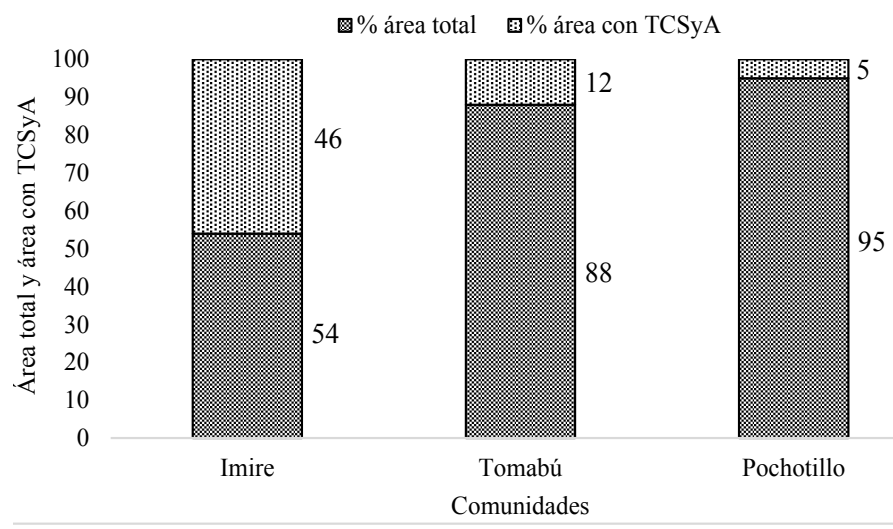

Figura 3. Área total de la finca y áreas con TCSyA.

Transferencia tecnológica (capacitación y asistencia técnica). Miranda y Ulloa (1993) afirman que el técnico debe conocer bien la innovación que promueve, para desarrollar confianza en sí mismo y formular argumentaciones convincentes a favor de la solución propuesta. En un proceso de transferencia la motivación nos hace actuar en tal o cual sentido, es el dispositivo que se activa para impulsar cualquier acción. No obstante existe una particularidad en las zonas de estudio; los temas de capacitación y las tecnologías que se transfieren a los productores, son seleccionados por los técnicos de las instituciones. Este hecho representa una limitante en los procesos de transferencia y adopción de tec nologías ya que este enfoque parte de una oferta tecnológica, y no de una verdadera demanda de parte de los productores, quienes quizás puedan o no Cuadro 4. Organizaciones, proyectos y servicios ofertados en las comunidades

\begin{tabular}{|c|c|c|c|}
\hline Comunidad & Organizaciones & Proyectos & Servicios \\
\hline $\begin{array}{l}\text { Los } \\
\text { Pochotillos }\end{array}$ & $\begin{array}{l}\text { Alcaldía de San Francisco Libre, } \\
\text { Instituto Nicaragüense de } \\
\text { Tecnología Agropecuaria } \\
\text { (INTA), Visión Mundial }\end{array}$ & $\begin{array}{l}\text { Implementación de sistemas } \\
\text { Agroforestales, reparación de } \\
\text { caminos, riego por gravedad }\end{array}$ & $\begin{array}{l}\text { Asistencia técnica, impulso de } \\
\text { proyectos }\end{array}$ \\
\hline Tomabú & $\begin{array}{l}\text { Alcaldía de La Trinidad, INTA, } \\
\text { Visión Mundial, FIDER, UNAG, } \\
\text { INTECFOR, PMA, Cáritas de } \\
\text { Nicaragua, ENACAL }\end{array}$ & $\begin{array}{l}\text { Implementación de sistemas } \\
\text { agroforestales más obras de } \\
\text { conservación de suelos y agua, } \\
\text { capacitación a mujeres, } \\
\text { préstamos, agua potable }\end{array}$ & $\begin{array}{l}\text { Asistencia técnica, impulso de } \\
\text { proyectos, ayuda de alimentos, } \\
\text { trabajo por comida, atienden } \\
\text { necesidades }\end{array}$ \\
\hline Imire & $\begin{array}{l}\text { Alcaldía de San José de } \\
\text { Cusmapa, Asociación "Padre } \\
\text { Fabretto", Comunidad Indígena, } \\
\text { Juan XXIII, INPRHU, UNICAM }\end{array}$ & $\begin{array}{l}\text { Producción de patios, salud } \\
\text { médica }\end{array}$ & $\begin{array}{l}\text { Asistencia técnica, impulso de } \\
\text { proyectos, apadrinamiento de } \\
\text { niños, colectivo de ventas de } \\
\text { potenciales artesanos }\end{array}$ \\
\hline
\end{tabular}
implementarlas.

Los métodos de transferencia empleados por las instituciones en estas comunidades son días de campo, fincas modelo, talleres, giras de intercambio y videos y reuniones, destacándose en las tres comunidades los días de campo y talleres (75-100\% de opinión). Esta combinación de teoría y práctica es determinante en la efectividad de la transferencia. En el caso de Los Pochotillos se registró en un $44 \%$ a las finca modeles como método de extensión y en un $47 \%$ a las giras de intercambio, mientras que en Tomabú e Imire se registra un bajo porcentaje (7-12\%) en cuanto a videos y reuniones y giras de intercambio.
Organización. La organización debe concebirse como un sistema abierto con múltiples relaciones, propósitos o funciones necesarias para integrar y coordinar. La organización conlleva muchos subsistemas e interacción dinámica, dado que los subsistemas son mutuamente dependientes, sus cambios afectarán el comportamiento de los demás, la organización existe en un ambiente dinámico, las múltiples relaciones entre la organización y su ambiente dificultan especificar de manera clara las fronteras de una entidad dada. Este conjunto de procesos, plenamente diferenciados e integrados en un sistema, es lo que define a la organización.

En estas comunidades se han trabajado organizadamente con organismos e instituciones desde los finales de la década de los años 90, con proyectos que han contribuido a la conservación del ambiente y los recursos naturales. La influencia de la organización en el proceso de adopción fue determinante. En el cuadro 4 se indican las principales organizaciones presentes en cada comunidad así como los proyectos y el tipo de servicios que brindan.

Los productores organizados presentaron una mayor tasa de adopción de prácticas de conservación de suelo y agua en comparación con los grupos no organizados y que trabajan de manera independiente y sin una perspectiva comunitaria y social. En el caso de Tomabú el 68\% de los productores se encuentran organizados y han logrado adoptar hasta 16 TCSyA contra ochos tecnologías que adoptan los no organizados. En el caso de Los Pochotillos el 91\% están organizados y adoptan ocho prácticas contra una tecnología adoptada por los no organizados. En Imire todos los productores se encuentran organizados y han adoptado hasta 15 tecnologías.

Seguridad alimentaria: disponibilidad de alimento. Las familias de las tres comunidades no logran garantizar la disponibilidad de los alimentos (cantidad suficiente) para el núcleo familiar debido a reducidas áreas agrícolas, bajos índices productivos, incidencia de plagas (cogollero, ratas, mosca blanca, langosta el pulgón, y comején), así como el 
efecto negativo de los excesos o ausencias de lluvias y la falta de capital (crédito) para la producción.

En las tres comunidades existen importantes áreas destinadas a la producción ganadera (figura 2). Estas áreas son utilizadas para la alimentación de las especies bovinas haciendo uso del sistema silvopastoril, división de potreros y cercas vivas con árboles de madero negro (Gliricidia sepium), Guácimo de ternero (Guásuma ulmifalia Lam) y Jícaro (Crescentia alata). Igualmente las áreas destinadas al bosque son importantes y son utilizadas en su mayoría con fines de regeneración natural y con diques para la retención del suelo.

En el caso de las áreas destinadas a la agricultura se evidenció bajos índices productivos en los principales cultivos (maíz, sorgo y frijol), sin embargo, en estos sistemas se implementa la incorporación de rastrojos, barreras muertas, barreras vivas, curvas a nivel, sistemas de granos básicos con árboles dispersos, diques, sistema de riego y cercas vivas.

Los índices productivos en aquellas áreas con $\mathrm{y}$ sin TCSyA, muestran leves diferencias debido principalmente a la ausencia de buenas prácticas agrícolas (figura 4). Los comunitarios de las zonas se abastecen de agua a través de ríos, pequeños pozos; excepto en Tomabú que lo hacen por servicio comunitario.

$\mathrm{Si}$ al problema de los bajos rendimientos se le adiciona la incidencia de plagas, variabilidad en las precipitaciones, y la falta de crédito para la producción, el escenario para los agricultores no es nada halagador y por ende no representan ninguna oportunidad para mejorar las condiciones de vida de las familias en los territorios.

Acceso a los alimentos. El elemento nivel y distribución de los ingresos familiares indica que estos productores no alcanzan a cubrir con sus ingresos, el costo de la canasta básica de alimentos. Con los ingresos económicos o margen bruto en la variable de ingresos productivos, se pretende demostrar si realmente los ingresos agropecuarios son mayores en aquellos productores que realizan manejo sostenible de suelo y ver qué efecto hay en aquellos que han adoptados mayor números de técnicas de conservación de suelo y agua y determinar si esos mismos ingresos logran acercarse o superar el indicador umbral de reproducción simple (URS) y poder identificar los niveles de pobreza en que se encuentran las familias.

Para determinar esté indicador socio económico se trabajó con el valor equivalente a dos canastas básicas rurales al mes, que alcanzan un valor anual de USD 2068.32 para una familia de seis miembros, valor que se estima en función del número de miembros que conforman las familias en cada territorio (FAO, 1991). Una vez estimado este valor para cada comunidad, se comparó con los ingresos económicos productivos promedios anuales.

En el cuadro 5 se observan los promedios por cada comunidad del margen bruto anual obtenido en la producción, la estimación del URS de acuerdo al número de miembros que conforman la familia, y se muestran estos ingresos por día y per cápita. Estos datos permiten hacer una comparación entre las comunidades para determinar la capacidad que tienen los productores de alcanzar con sus ingresos económicos o margen bruto obtenido en la producción el umbral de reproducción simple.

En la figura 5 se muestra el comportamiento del margen bruto o ingresos económicos productivos en las tres comunidades. Solamente la comunidad de Tomabú, que ha adoptado mayor número de tecnologías logra con sus ingresos promedios anuales de USD 3288.00 superar en casi el doble (194\%) el umbral económico de USD 1 805.00, necesario para satisfacer sus necesidades. En Los Pochotillos los

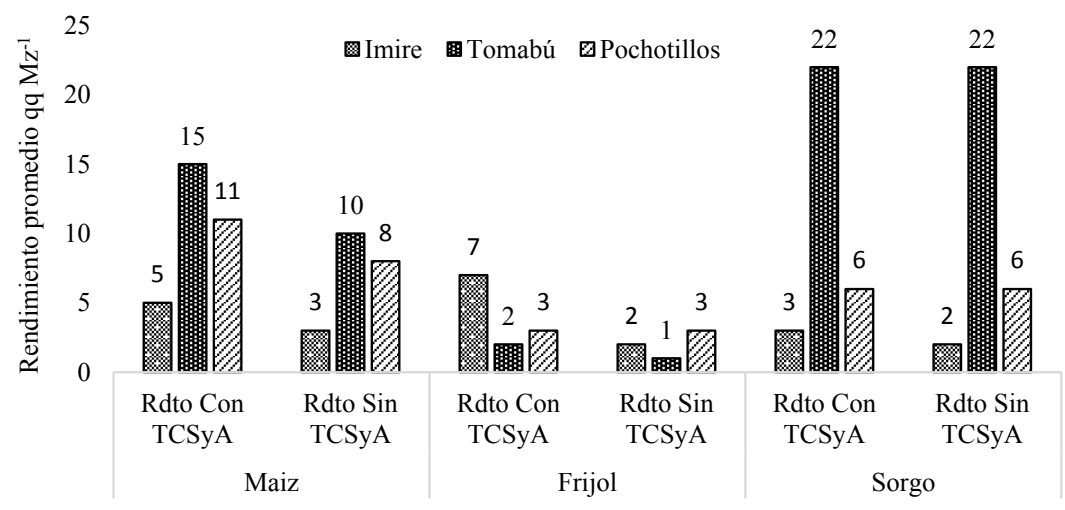

Figura 4. Rendimiento promedio de granos básicos por comunidad ciclo 2008-2009 en áreas con y sin TCSyA 
Cuadro 5. Disponibilidad promedio de Ingresos por familia por día y por comunidad

\begin{tabular}{lccccc}
\hline Comunidad & $\begin{array}{c}\text { Promedio de } \\
\text { margen bruto } \\
\text { anual (USD) }\end{array}$ & $\begin{array}{c}\text { URS estimado } \\
\text { anual (USD) }\end{array}$ & $\begin{array}{c}\text { Promedio de } \\
\text { miembros } \\
\text { de la familia }\end{array}$ & $\begin{array}{c}\text { Promedio de } \\
\text { ingresos por día }\end{array}$ & $\begin{array}{c}\text { Per } \\
\text { cápita/día }\end{array}$ \\
\hline Los Pochotillos & 432.00 & 2063.00 & 6 & 1.18 & 0.19 \\
Tomabú & 3288.00 & 1805.00 & 5 & 9.01 & 1.68 \\
Imire & 255.00 & 1724.00 & 5 & 0.70 & 0.14 \\
\hline
\end{tabular}

URS: Umbral de reproducción simple

ingresos promedios anuales de USD 432.00 apenas alcanzan el 30\% de su umbral económico (USD 2 063.00), por su parte los productores de Imire con sus ingresos promedios anuales de USD 255.00 alcanzan apenas el 19\% del umbral económico (USD 1 724.00).

Incidencia de la adopción en la seguridad alimentaria. En lo que respecta a Seguridad Alimentaria, las familias de las tres comunidades tienen incapacidad de acceder económicamente a los alimentos disponibles. Los niveles de ingresos familiares logrados no cubren el precio de la canasta básica, lo que los hace incapaz de acceder económicamente a los alimentos disponibles, teniendo una relación directa con el mapa de pobreza extrema a nivel nacional.

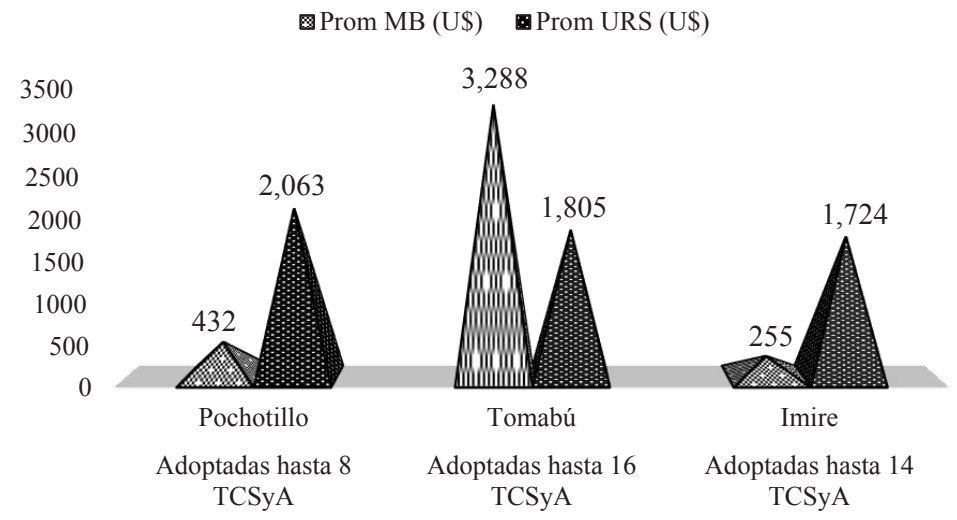

Figura 5. Margen bruto productivo anual y umbral de reproducción simple de productores por comunidad.

Las comunidades de Los Pochotillos e Imire, reflejan alarmantes datos de ingresos productivos promedios e ingresos per cápita, los resultados encontrados fueron; Para la comunidad de Imire apenas logran USD0.70 por día para una familia promedio de cinco miembros, reflejando un perca pita de USD 0.14, de USD 1.18 y USD 0.19 para Los Pochotillos y de 9.01 y 1.68 para Tomabú.

Las comunidades de Imire y Los Pochotillos se clasificarían en pobreza absoluta puesto que sus ingresos per cápita son inferiores a un dólar por día. En el caso de Tomabú se considera que viven en condiciones de pobreza por obtener ingresos inferiores a dos dólares per cápita por día.

Referente a la disponibilidad de alimento (producción local de granos básicos) se observa un efecto de leve mejoría en los rendimientos de maíz y frijol en áreas productivas con TCSyA en relación a áreas sin tecnologías, pero no logran con esto, satisfacer las necesidades reales de las familias.

Finalmente podemos concluir que el efecto de las tecnologías en materia de conservación del suelo aún no ha sido suficiente para incrementar los ingresos productivos y el aseguramiento de los alimentos a las familias en las comunidades en estudio.

\section{CONCLUSIONES}

Los productores de las tres comunidades adoptan técnicas de conservación de suelo y agua transferidas por las instituciones de desarrollo, además poseen una percepción favorable de las innovaciones que les han sido transferidas; no obstante, no logran incrementar los rendimientos de sus cultivos, lo cual no les permite lograr la seguridad alimentaria, mejorar sus condiciones de vida y alcanzar un desarrollo a nivel local.

Se confirma que el proceso de adopción de tecnologías es dinámico y complejo, y las estrategias de intervención implementadas por las instituciones de desarrollo influyen en la adopción de las tecnologías transferidas.

La tenencia de la tierra es diferente en las tres comunidades, existiendo un porcentaje altos de tenencia de la tierra del tipo propia, lo que permite que los productores adopten las tecnologías transferidas y dispongan de las condiciones para el aseguramiento de la producción de alimentos para las familias.

La adopción de tecnologías es independiente del nivel de escolaridad en las tres comunidades, ya que se manifestó que sin tener un nivel de educación, los productores adoptan tecnologías.

Las capacitaciones y la transferencia de tecnologías son seleccionadas por los técnicos, esto dificulta la apropiación de dichas técnicas por los productores y sus familias.

Fue evidente que el factor organización es determinante ya que se destacan los productores organizados adoptando mayor número de tecnologías.

Independientemente de los niveles de ingresos productivos alcanzado por las familias en estas comunidades, los productores adoptan las tecnologías. 


\section{REFERENCIAS BIBLIOGRÁFICAS}

ATyV; PASOLAC (Asociación Tierra y Vida), (Programa para la Agricultura Sostenible en Laderas de América Central). 1999. Herramientas socioeconómicas de seguimiento y evaluación de la transferencia en CSyA. Editorial Ciencias Sociales, Managua, NI. 171 p.

CIMMYT (Centro Internacional de Mejoramiento de Maíz y Trigo, MX). 1993. La adopción de tecnologías agrícolas: guía para el diseño de encuestas. Programa de economía del CIMMYT, MX, DF. 88 p.

IICA (Instituto Interamericano de Cooperación para la Agricultura, NI). 2005. Inventario de políticas agroambientales en Nicaragua, descripción de los principales problemas ambientales relacionado con la agricultura. Managua NI. P 8.

INIDE (Instituto Nicaragüense de Estadísticas). 2005. VIII Censo de Población y IV de Vivienda. (en línea). Consultado 2 mar. 2015. Disponible en http:/www.inide.gob.ni/censos2005/VolPoblacion/Volumen\%20Poblacion\%201-4/Vol.IV\%20PoblacionMunicipios.pdf

INTA (Instituto Nicaragüense de Tecnología Agropecuaria). 2006. Informes de campo de técnicos, filial municipio de La Trinidad, Departamento de Estelí.

Miranda y Ulloa. 1993. Transferencia de tecnologías para el desarrollo rural, retos, problemas y perspectivas. Programa de la Agricultura Sostenible en Laderas de América Central (PASOLAC). 46 p.

Nowak, P. 1992. Why farmers adopt production technology. Journal of Soil and Water Conservation 47(1):14-16.

FAO (Organización de las Naciones Unidas para la Agricultura y la Alimentación). 1991. Diagnósticos de sistemas agrarios, una metodología operativa y tres estudios de casos en Chile, Santiago, CL. 170 p.

PRESANCA (Programa Regional de Seguridad Alimentaria y Nutricional para Centroamérica). 2009. Municipio de San José de Cusmapa, departamento de Madriz, Nicaragua. Fortalecimiento municipal en seguridad alimentaria y nutricional y desarrollo local 2006-2009. Resumen de Logros y Avances. 54 p.

Radulovich, F. (1999). La Adopción de Tecnologías. La Perspectiva del Agricultor y sus implicaciones para la elaboración de Políticas. 1 - 3 de diciembre de 1997, San José, CR.

Ramírez, R; Shultz, S; Hearne, R; Gómez, M. 2000. Conteo de Poisson: modelos econométricos para explicar la adopción de tecnologías agrícolas por pequeños productores en El Salvador y Panamá. Revista Forestal Centroamericana 32:13-19.

Rogers; E. 1995. Difussión of innovations. 4 ed. New York, US. The Free Press. 519 p. 